\title{
An optimized ink-reducing hollowed-out arm meander dipole antenna structure for printed RFID tags
}

\author{
ADI M. J. MARINDRA, PORNANONG PONGPAIBOOL, WERAYUTH WALLADA \\ AND SIWARUK SIWAMOGSATHAM
}

\begin{abstract}
This paper presents an optimized ink-reducing meander dipole antenna structure suitable for implementing printed radio frequency identification (RFID) tags. The proposed antenna designs contain empty ink-reducing hollowed-out areas along the antenna's arms such that the resulting antennas require much less conductive ink to produce yet still achieve decent antenna performance compared with the conventional solid-arm dipole antennas. The simulation results demonstrate that when the ratio between the width of the hollowed-out areas and the width of the antenna arms is about o.6, the resulting RFID tag experiences a slight read range performance degradation of $<10 \%$, while it offers a sizeable ink consumption reduction of almost $50 \%$.
\end{abstract}

Keywords: Printed RFID, Printed antenna, Ink-reducing antenna, Hollowed-out arm

Received 31 March 2015; Revised 20 November 2015; Accepted 23 November 2015; first published online 7 January 2016

\section{INTRDDUCTION}

Printed radio frequency identification (RFID) technology has recently gained much interest for item-level labeling and tracking applications. Printed RFID tags are simpler, more economical, and more environmental friendly to produce than RFID tags fabricated by the traditional copper-etching process $[1,2]$. By utilizing a low-cost printing technique such as inkjet printing or screen printing, a printed RFID tag antenna of a desirable planar structure can be economically formed such that conductive ink is only printed on the intended areas and no ink is wasted in the unused nonconductive areas [3-7]. Nonetheless, design challenges exist due to the low conductivity characteristic and the relatively high price of conductive ink materials. In general, a costeffective printed RFID tag antenna design should minimize the amount of conductive ink required for printing a designed tag antenna structure, while the resulting RFID tag should still achieve good read range performance.

Over recent years, many researchers have proposed various printed RFID tag antenna designs that require less conductive ink to produce, e.g. [8-14]. In [8], a grid pattern is employed for antenna structures with large conductive areas such as tip-loaded dipole antennas, but the results demonstrate that forming grid patterns on small tag antennas greatly degrades

National Electronics and Computer Technology Center 112 Thailand Science Park, Phahonyothin Road, Khlong Nueng, Khlong Luang, Pathum Thani 12120, Thailand. Phone: +6625646900

Corresponding author:

P. Pongpaibool

Email: pornanong.pongpaibool@nectec.or.th the antenna performance. In [9, 10], a narrow-line RFID dipole antenna structure is proposed, but the resulting design achieves low antenna gain and poor read range performance. In $[11-13]$, the thickness of conductive layers in different areas of the antenna is selectively optimized. This approach yields quite good results; however, the reprinting process to formulate variable levels of conductive layer thickness is quite complicated and time consuming. In [14], an ink-optimized slotted bow-tie antenna design is introduced for a printed RFID tag antenna. However, the gain of this antenna is noticeably reduced compared with the solid-arm bow-tie antenna design.

This work proposes an ink-reducing meander dipole antenna structure that requires less conductive ink for fabrication of good performance low-cost RFID tags. In the proposed antenna structure, a meandering empty hollowed-out area is formed along the centerline of each arm of the meander dipole antenna in order to decrease ink consumption. The simulation results demonstrate that the proposed ink-reducing meander dipole antenna structure can trim down the amount of conductive ink required to produce the resulting tag by about $46 \%$, while the resulting printed RFID tag still achieves a comparable level of read range performance as the printed RFID tag composed of a solid-arm dipole antenna.

\section{BACKGRDUND}

\section{A) Printed RFID tags and performance criteria}

Basically, a printed RFID tag consists of two main components: a printed antenna and an RFID chip attached to the 
antenna. A dipole-type antenna structure is usually employed since it produces a desirable omni-directional radiation pattern. A simple dipole antenna structure for printed RFID tags consists of two radiating arms and a matching section formed by an inductive shorting loop connecting the two radiating arms. The inductive matching section should be properly designed to have good matching with the impedance of the attached RFID chip. The antenna is usually printed using conductive ink on a flexible substrate such as polyethylene-terephthalate (PET).

The performance of RFID tags is normally illustrated by the read range $(r)$ which can be calculated using Frii's transmission formula as [15]

$$
r=\frac{\lambda}{4 \pi} \sqrt{\frac{g_{t} g_{r} P_{t}}{P_{t h}} p\left(1-\left|s_{11}\right|^{2}\right)},
$$

where $\lambda$ is the wavelength, $P_{t}$ is the power transmitted by the RFID reader, $g_{t}$ denotes the gain of the transmitting antenna, $g_{r}$ denotes the gain of the receiving tag antenna, $P_{t h}$ denotes the threshold power for the RFID chip to operate, $p$ denotes the polarization loss factor, and $\left|s_{11}\right|^{2}$ corresponds to the Kurokawa's power reflection coefficient [16]. In Thailand, RFID tags must operate within the frequency range of 920$925 \mathrm{MHz}$ in the ultra-high-frequency band. In addition, the maximum level of $g_{t} P_{t}$ must not exceed $4 \mathrm{~W}$.

Here, the reflection coefficient $\left(s_{11}\right)$ indicates how well a given tag antenna structure is matched to the attached RFID chip. The reflection coefficient $\left(s_{11}\right)$ can be obtained by

$$
s_{11}=\frac{Z_{a}-Z_{c}^{*}}{Z_{a}+Z_{c}},
$$

where $Z_{a}=R_{a}+j X_{a}$ denotes the antenna impedance, and $Z_{c}=R_{c}+j X_{c}$ denotes the chip impedance. The antenna and the RFID chip are well matched if the antenna impedance $\left(Z_{a}\right)$ is equal to the conjugate value of the chip impedance $\left(Z_{c}\right)$. The perfect conjugate impedance matching condition is desirable for good designs.

\section{B) Straight and meander dipole antennas for printed RFID tags}

A straight dipole antenna is a basic form of dipole antenna design. A drawback of using a straight dipole antenna for RFID tags is its length. A straight dipole antenna must be long enough $(\approx 0.5 \lambda)$ to achieve the theoretical antenna gain of about $2.15 \mathrm{~dB}$ predicted for a half-wavelength dipole [17]. Otherwise, a straight dipole antenna with shorter arms $(\ll 0.5 \lambda)$ has a degraded antenna gain. A long, straight dipole antenna is not desirable in practice since small-sized tags are typically required for RFID applications.

To miniaturize the lengthy straight dipole antennas, meander dipole antenna designs have been widely used [18, 19]. Instead of having long straight arms, the arms of a meander dipole antenna meander back and forth, so that the RFID tag becomes shorter in length. Still, the meandered antenna arms can be effectively long enough to yield a similar antenna gain as that of the straight dipole antenna. As an illustration, two simple straight dipole antenna designs with different antenna dimensions and a meander dipole antenna design are presented and compared. Figures $1(\mathrm{a})$ and 1 (b) show the basic straight dipole antenna designs with a tag length of $118 \mathrm{~mm}(\approx 0.36 \lambda)$ and $82 \mathrm{~mm}\left(\approx_{0.25} \lambda\right)$, respectively. Figure $1(\mathrm{c})$ displays the meander dipole antenna design in which the antenna arms are fitted onto an $82 \times 10 \mathrm{~mm}^{2}$ tag. Table 1 lists the characteristic parameters of the employed antenna substrate, conductive materials, and the RFID chip. Table 2 presents the simulation results obtained via the CST Microwave Studio 2014 simulation software. It can be seen that the long $0.36 \lambda$ straight dipole antenna design can achieve a read range of about $7 \mathrm{~m}$, while the shorter $0.25 \lambda$ straight dipole antenna design can only achieve a read range of $<_{3} \mathrm{~m}$. In contrast, the meander dipole antenna design with a tag length of $0.25 \lambda$ achieves a read range of about $5.3 \mathrm{~m}$, which is noticeably larger than the read range of the short $0.25 \lambda$ straight dipole antenna design. Nonetheless, the standard meander dipole antenna design should be reengineered, so that the resulting tag antenna does not only have a short overall tag length, but also requires less conductive ink.

\section{INK-REDUCING DIPDLE ANTENNA DESIGN}

This section presents an optimized ink-reducing dipole antenna structure for low-cost printed RFID tags. In the proposed design, the conventional dipole antenna configurations depicted in Figs $1(\mathrm{a})$ and 1 (c) are modified such that some meandering hollowed-out areas are formed along the centerline of the antenna arms, and no conductive ink is filled in the empty areas. The resulting tag antennas are displayed in Figs 2(a) and 2(b), wherein $W$ denotes the width of the antenna arms and $W_{\text {space }}$ denotes the width of the empty areas. Ink consumption is lower when $W_{\text {space }}$ is widened or the ratio between $W_{\text {space }}$ and $W$ is increased. The empty areas are only formed along the antenna arms, while the inductive matching loop section remains fully filled with conductive ink.

Note that ideally for each design with a given value of $W_{\text {space }}$, the optimal matching loop section may not be identical. The matching loop section adjustments are numerically studied in this work but the results suggest that the presented fixed matching loop section with a constrained line width can approximately achieve optimum impedance matching for all values of $W_{\text {space }} / W$ under consideration. Furthermore, if the matching loop section is also hollowed out, the antenna gain performance drastically degrades.

\section{SIMULATION RESULTS}

This section presents the simulation results obtained from the CST Microwave Studio 2014 simulation tool. The simulation employs the characteristic parameters of the substrate, conductive ink, and RFID chip as listed in Table 1. The read range performance is calculated using equation (1) with $g_{t} P_{t}=4 \mathrm{~W}$ and the polarization loss factor $p=1$.

Figure 3 illustrates the performance results of the inkreducing $0.36 \lambda$ straight dipole antenna designs as a function of $W_{\text {space }} / W$. The conventional antenna design with fully filled arms corresponds to the case when $W_{\text {space }} / W=0$. It can be observed that enlarging the empty areas along the arms of the straight dipole antenna decreases the antenna gain and increases the reflection coefficient. Consequently, the read range results are poorer when the unfilled areas are 


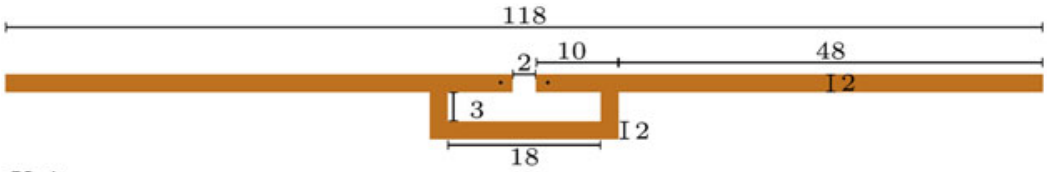

Unit: $\mathrm{mm}$

(a)

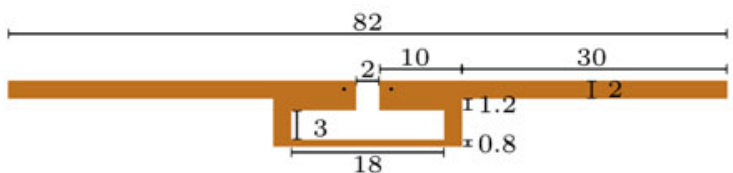

Unit: mm

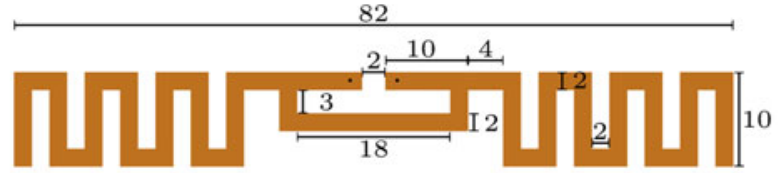

Unit: mm

(c)

Fig. 1. Illustration of dipole antenna miniaturization with meander line structure: (a) a straight dipole antenna with a total length of $0.36 \lambda$, (b) a short straight dipole antenna with a total length of $0.25 \lambda$, and (c) a meander dipole antenna with a total length of $0.25 \lambda$.

Table 1. Characteristic parameters of the employed substrate, conductive ink, and RFID chip.

\begin{tabular}{lll}
\hline Material & Parameter & Value \\
\hline PET film & Thickness & $135 \mu \mathrm{m}$ \\
& Relative permittivity & 3 \\
& Loss tangent & 0.002 \\
Conductive ink & Conductive layer thickness & $1 \mu \mathrm{m}$ \\
& Volume resistivity & $10 \times 10^{-6} \Omega \mathrm{cm}$ \\
RFID chip & Model & $\mathrm{NXP} \mathrm{UCODE} \mathrm{G2XL}$ \\
& Input capacitance & $1.36 \mathrm{pF}$ \\
& Quality factor & 9 \\
& Minimum operating power & $-15 \mathrm{dBm}$ \\
& $\left(P_{\text {th }}\right)$ & \\
& Impedance at $922.5 \mathrm{MHz}$ & $14.095-j 126.857 \Omega$ \\
\hline
\end{tabular}

Table 2. Calculated results of the dipole antenna designs at $922.5 \mathrm{MHz}$, with $p=1$ and $g_{t} P_{t}=4 \mathrm{~W}$.

\begin{tabular}{lllll}
\hline Antenna & $\begin{array}{l}\text { Antenna gain } \\
(\mathbf{d B})\end{array}$ & $\begin{array}{l}S_{11} \\
(\mathbf{d B})\end{array}$ & $\begin{array}{l}\text { Read } \\
\text { range }(\mathbf{m})\end{array}$ & $\begin{array}{l}\text { Ink volume } \\
\left(\mathbf{m m}^{3}\right)\end{array}$ \\
\hline $\begin{array}{c}0.36 \lambda \text { straight } \\
\text { dipole }\end{array}$ & -2.32 & -33.9 & 7.01 & 0.288 \\
$\begin{array}{c}0.25 \lambda \text { straight } \\
\text { dipole }\end{array}$ & -10.0 & -26.2 & 2.88 & 0.209 \\
$\begin{array}{c}\text { 0.25 meander } \\
\text { dipole }\end{array}$ & -4.74 & -30.8 & 5.63 & 0.428 \\
\hline
\end{tabular}

widened. For instance, the read range of the ink-reducing $0.36 \lambda$ straight dipole antenna with a $W_{\text {space }} / W$ ratio of 0.6 is $6.5 \mathrm{~m}$, which is only $0.5 \mathrm{~m}$ shorter than the read range of the conventional $0.36 \lambda$ straight dipole antenna with fully filled arms. That is, the proposed design achieves an ink reduction of nearly $40 \%$, while the read range is only $7 \%$ shorter than that of the conventional $0.36 \lambda$ straight dipole antenna design.

Figure 4 displays the performance results of the proposed ink-reducing $0.25 \lambda$ meander dipole antenna designs as a

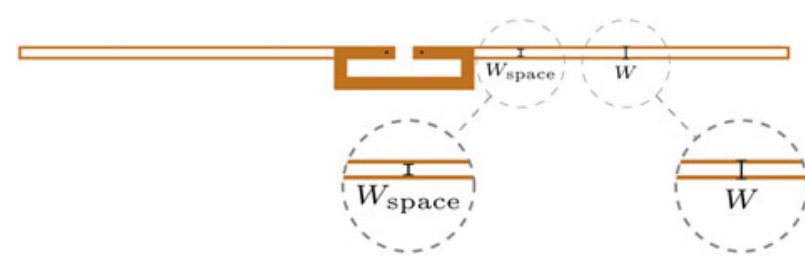

(a)

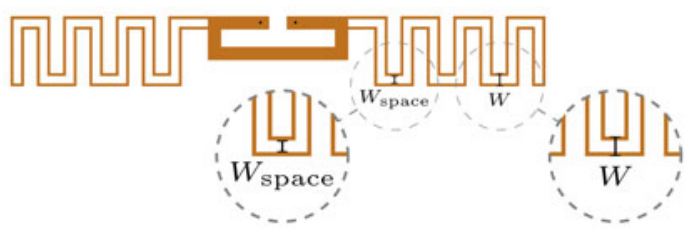

(b)

Fig. 2. Ink-reducing dipole antenna designs: (a) ink-reducing $0.36 \lambda$ straight dipole antenna and (b) ink-reducing $0.25 \lambda$ meander dipole antenna.

function of $W_{\text {space }} / W$. Similarly, the read range results are poorer when the unfilled areas are widened. For example, the read range of the ink-reducing $0.25 \lambda$ meander dipole antenna with a $W_{\text {space }} / W$ ratio of 0.6 is $5.27 \mathrm{~m}$, which is only $0.36 \mathrm{~m}$ shorter than the read range of the conventional $0.25 \lambda$ meander dipole antenna. That is, the proposed design achieves an ink reduction of $46 \%$, while the read range is only $6 \%$ shorter than that of the conventional $0.25 \lambda$ meander dipole antenna design.

In addition, the surface current density distribution of the straight and meander dipole antenna configurations are illustrated in Fig. 5. It can be noted from Figs 5(a) and 5(c) that the current density along the centerline of the fully filled antenna arms is relatively small. Therefore, it may be predicted that introducing some narrow, ink-reducing hollowed-out areas along the centerline of the printed dipole arms should have little impact on the antenna radiation performance, according to the design concept suggested in [13]. Moreover, it may be observed from Figs 5 (b) and 5 (d) that the in-phase current density for the proposed hollowed-out 


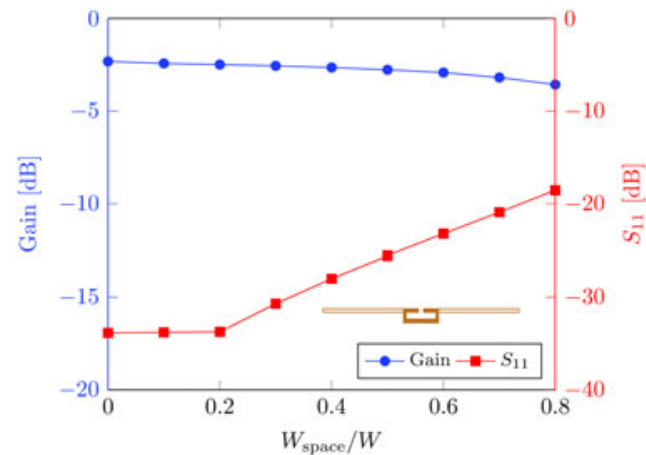

(a)

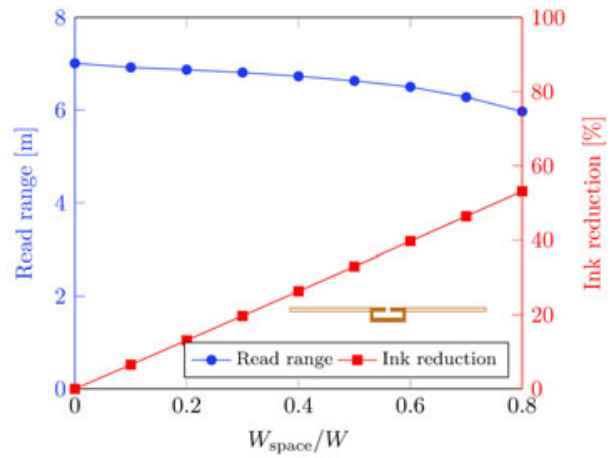

(b)

Fig. 3. Performance results of the ink-reducing $0.36 \lambda$ straight dipole antenna: (a) antenna gain and $S_{11}$ and (b) read range and ink reduction, as a function of $W_{\text {space }} / W$.

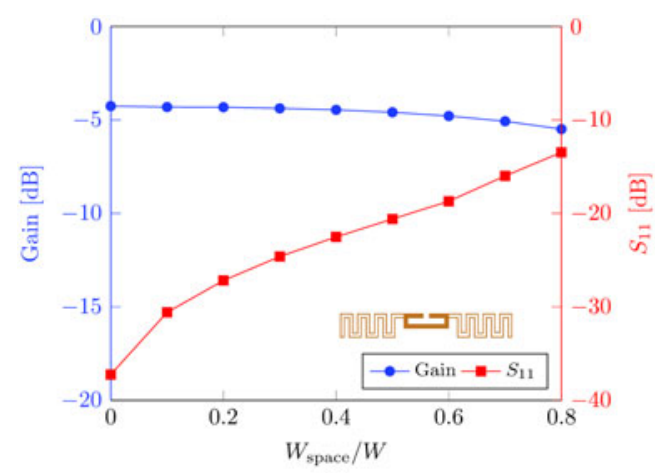

(a)

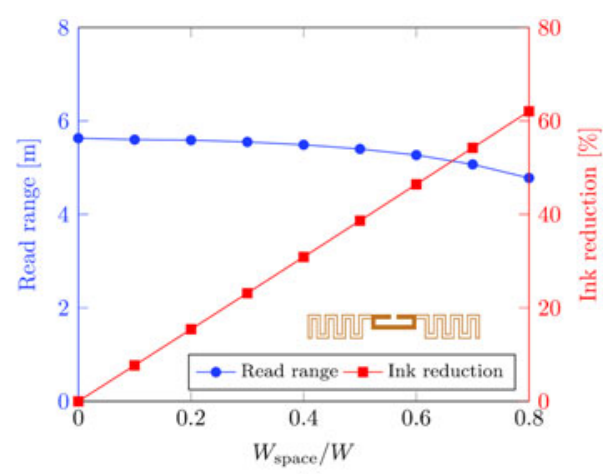

(b)

Fig. 4. Performance results of the ink-reducing $0.25 \lambda$ meander dipole antenna: (a) antenna gain and $S_{11}$ and (b) read range and ink reduction, as a function of $W_{\text {space }} / W$.

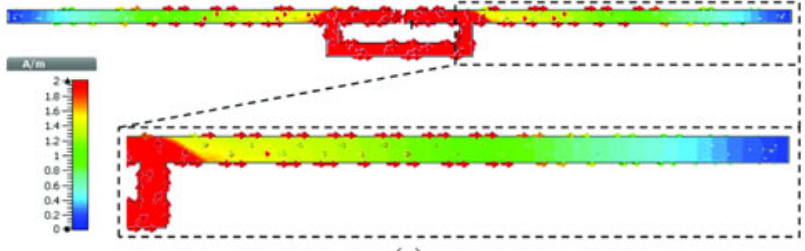

(a)

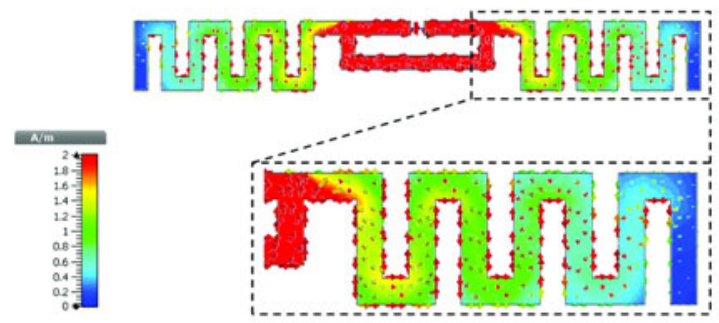

(c)

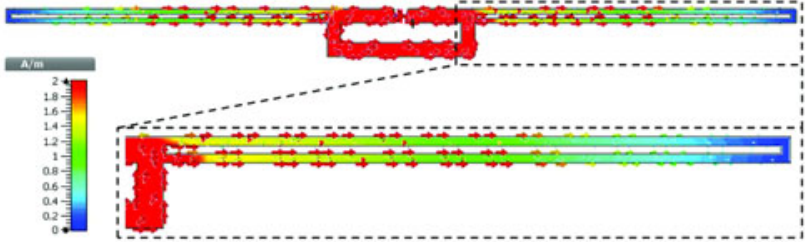

(b)

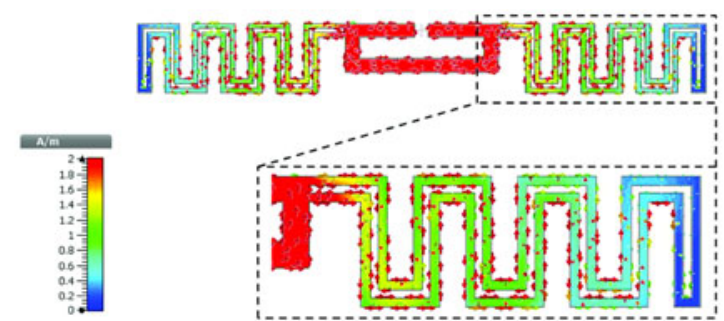

(d)

Fig. 5. Surface current density distribution at $922.5 \mathrm{MHz}$ (a) the $0.36 \lambda$ straight dipole antenna, (b) the ink-reducing o.36 $\lambda$ straight dipole antenna with $W_{\text {space }}$ $W=0.3$, (c) the $0.25 \lambda$ meander dipole antenna, and (d) the ink-reducing $0.25 \lambda$ meander dipole antenna with $W_{\text {space }} / W=0.3$.

arm dipole antennas is higher along the edge of the antenna arms compared with that of conventional dipole counterparts. Possibly, the effect of the in-phase current density increase along the edge areas may well compensate for the effect of the current absence over the hollowed-out areas. Depending on the surface current density distribution for each part of the entire antenna structure, the resulting antenna gain of the hollowed-out arm antenna designs may be similar to or worse than that of the conventional fully filled dipole antenna designs.

Furthermore, it is demonstrated here that the empty unfilled areas should only be formed on the antenna arms and the inductive matching section should remain fully filled. Figure 6 shows the performance results of the 


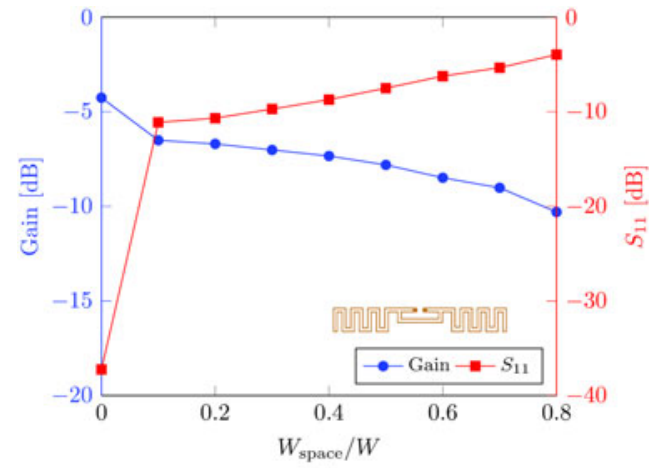

(a)

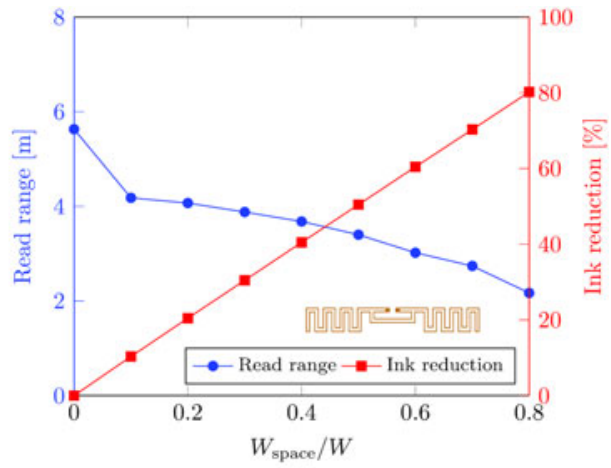

(b)

Fig. 6. Performance results of the ink-reducing $0.25 \lambda$ meander dipole antenna design in which the empty areas are extended over the inductive matching loop section: (a) antenna gain and $S_{11}$ and (b) read range and ink reduction, as a function of $W_{\text {space }} / W$.

ink-reducing $0.25 \lambda$ meander dipole antenna designs with the empty areas extended over the entire antenna body including the inductive matching part. In this case, the sizes of the hollowed-out matching loop section are also adjusted to improve impedance matching for each design. Nonetheless, the adjustments only yield slim improvements of the impedance matching quality given that the dimensions of the antenna arms are not altered. It can be seen that the presence of empty areas of any size over the inductive matching loop section largely increases the reflection coefficient and noticeably degrades the antenna gain and read range performance of the resulting design. In fact, this result is not surprising since the matching section is formed to adjust impedance of the antenna. A modification to this matching structure would cause a large impedance mismatch. Moreover, as shown in Fig. 5, the surface current density over the matching section of the dipole antenna is very dominant. According to the design concept presented in [13], this area should not be miniaturized in order to achieve good antenna gain performance.

\section{EXPERIMENTAL VERIFICATION}

\section{B) Fabrication of RFID tag antennas with inkjet printing}

To verify the simulation results, the presented antenna designs are fabricated by inkjet printing. The Mitsubishi NBSIJ-MUo1 silver ink and the Brother MFC-J825DW inkjet printer are used. The employed substrate is the Mitsubishi NB-TB${ }_{3} \mathrm{GU} 100$ PET sheet. According to the datasheet, the chosen silver ink can form a conductive layer that has thickness of approximately $1 \mu \mathrm{m}$ on the employed substrate.

To create an RFID tag, an RFID chip is attached to each antenna using conductive tapes. The chip has $3 \times 3 \mathrm{~mm}^{2}$ straps which are placed on the antenna feed points. Figure 7 displays the fabricated RFID tags. Both conventional and ink-reducing designs for the $0.36 \lambda$ straight dipole antenna and the $0.25 \lambda$ meander dipole antenna are fabricated. Here, the proposed ink-reducing designs employ the $W_{\text {space }} / W$ ratio of 0.6 as in the previous section.

It should be pointed out that the tag fabrication here is only intended for the purpose of verifying the proposed inkreducing antenna design approach. Although the fabricated RFID tags can be bent or twisted during our tests, additional

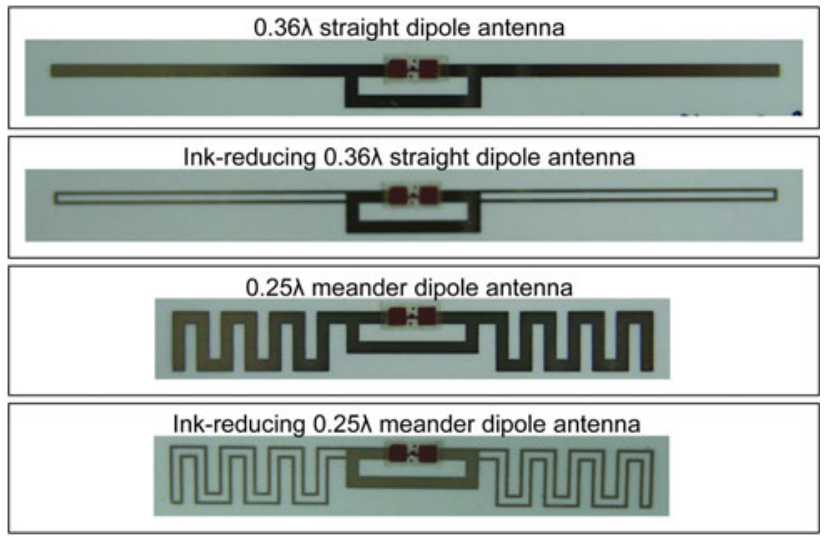

Fig. 7. Fabricated inkjet-printed RFID tag antennas.

coatings may be needed to reinforce the tags before they can really be deployed in practice. Additional studies on the issue of coating for RFID tags or wearable antennas may be found in $[20,21]$.

\section{B) Impedance measurement}

Since dipole-type RFID tags are categorized as balanced devices, the impedance cannot be measured directly using a measurement instrument that is terminated with unbalanced ports such as coaxial ports. When the impedance of a dipole antenna is characterized with a conventional method that uses a vector network analyzer (VNA) with the single-port measurement, the result will not be accurate because the currents fed to the radiators of the antenna are unequal.

In this paper, we use the method suggested in [22-24] which is reportedly accurate for impedance measurement of asymmetrical/symmetrical tag antennas. This measurement method uses a two-port VNA and a test fixture. As shown in Fig. 8(a), the test fixture here has an arm length of approximately $40 \mathrm{~mm}$. At one end, the test fixture is formed with two SMA connectors which can be connected to the VNA via some test cables. At the other end, the test fixture is formed such that the outer conductors of the coaxial cables are soldered together and the tip of the inner conductor of each coaxial cable is attached to a small $1 \times 1 \mathrm{~mm}^{2}$ copper pad that is used as a measurement end point. 


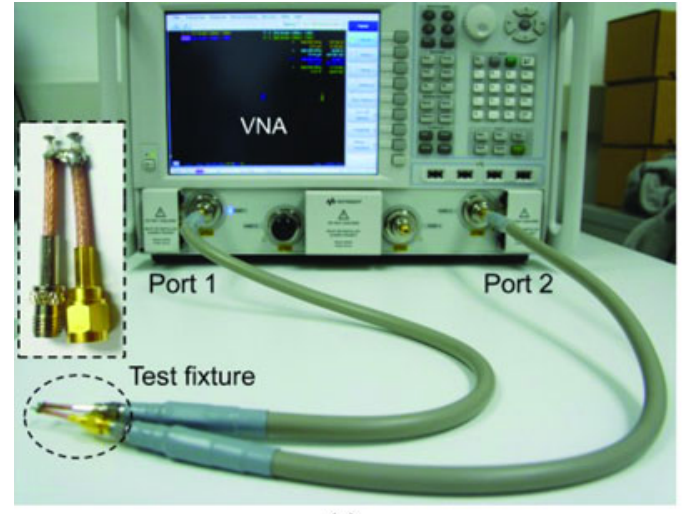

(a)

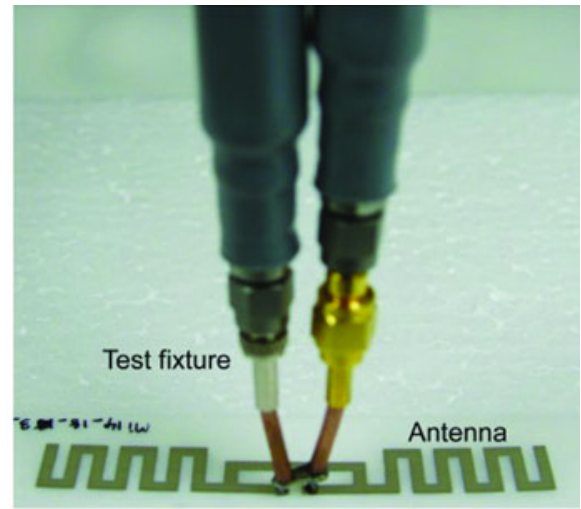

(b)

Fig. 8. Impedance measurements: (a) the measurement setup and (b) an impedance measurement of a printed antenna.

The Keysight PNA-X N5245A VNA is employed. After the standard two-port calibration is performed, the test fixture is connected to the coaxial test cables. The built-in automatic port extension feature is then used to offset the delay effect caused by the test fixture [23]. Here, the delay compensation offsets for the extended ports are 173 and 169 ps. Given that the velocity factor of the coaxial cables is approximately 0.7 , the obtained port extension delay offsets are equivalent to 36.3 and $35.5 \mathrm{~mm}$ in the distance domain, respectively. Note that these offset values are roughly equal to the arm length of the test fixture.

To measure the impedance of a given antenna, the endpoints of the test fixture are placed on the feed areas of the antenna as shown in Fig. 8(b). The measured two-port $S$-parameters, i.e. $\hat{s}_{11}, \hat{s}_{12}, \hat{s}_{21}$, and $\hat{s}_{22}$ are recorded from the VNA. Then, the differential impedance $\left(Z_{d}\right)$ of the antenna can be derived from the measured $S$-parameters by [23]

$$
Z_{d}=\frac{2 Z_{0}\left(1-\hat{s}_{11} \hat{s}_{22}+\hat{s}_{12} \hat{s}_{21}-\hat{s}_{12}-\hat{s}_{21}\right)}{\left(1-\hat{s}_{11}\right)\left(1-\hat{s}_{22}\right)-\hat{s}_{21} \hat{s}_{12}},
$$

where $Z_{\mathrm{o}}$ is the characteristic impedance of the system, which is equal to $50 \Omega$ for most measurement systems.

Figure 9 presents the comparison between the calculated and measured impedance of the fabricated tag antennas. It

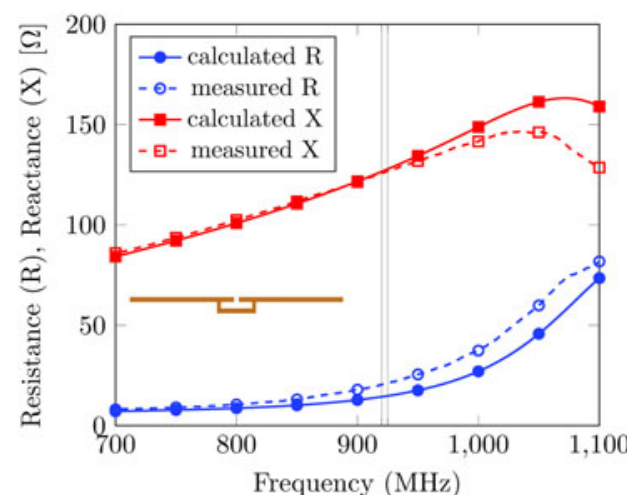

(a)

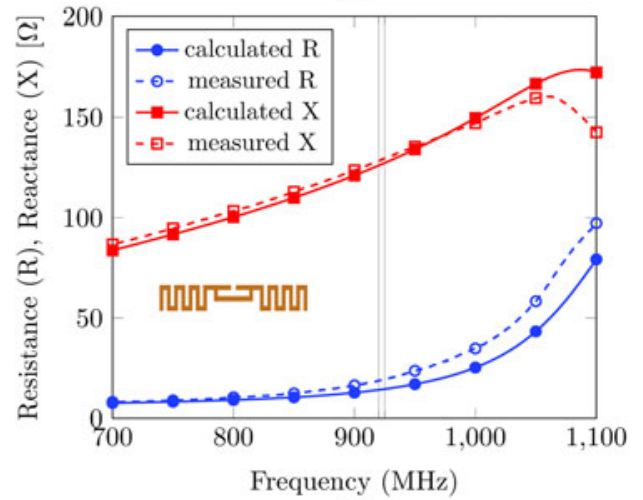

(c)

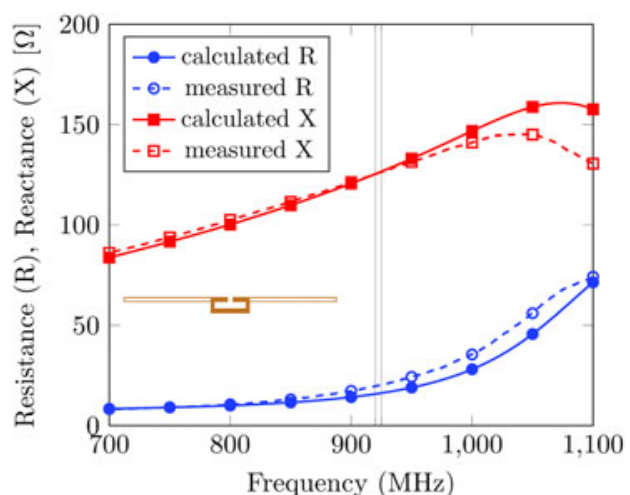

(b)

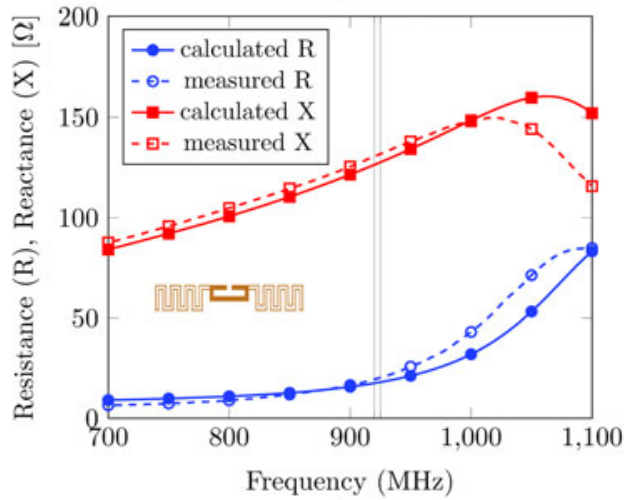

(d)

Fig. 9. Calculated and measured impedance results of the fabricated tag antennas: (a) the $0.36 \lambda$ straight dipole antenna, (b) the ink-reducing $0.36 \lambda$ straight dipole antenna, (c) the $0.25 \lambda$ meander dipole antenna, and (d) the ink-reducing $0.25 \lambda$ meander dipole antenna. 


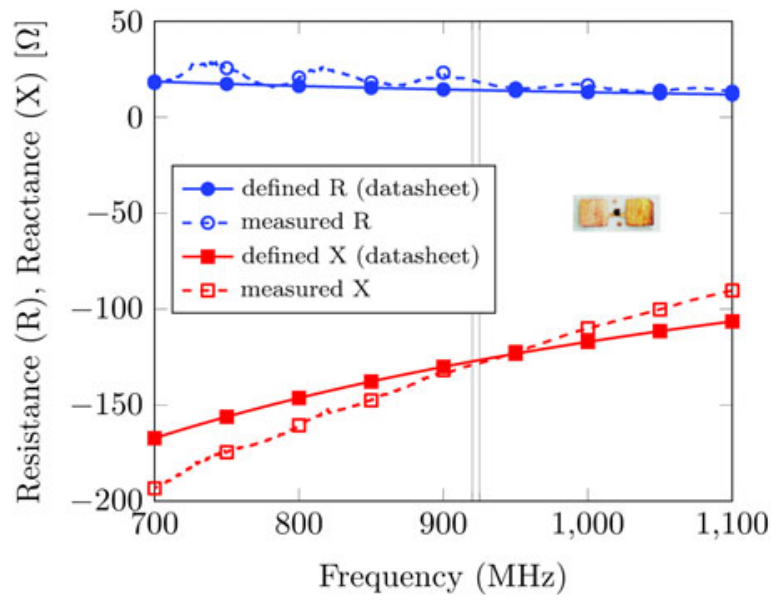

Fig. 10. Measured and defined impedance data of the employed RFID chip.

can be observed that the measured impedance data of the straight dipole antenna and the meander dipole antenna are fairly consistent with the calculated results. Figure 10 illustrates the impedance measurement results and the defined impedance data obtained from the datasheet of the employed RFID chip. The measured resistance results agree quite well with the defined resistance data. However, the differences between the measured and defined reactance results are larger at lower and higher frequencies. To some extent, the deviations may be caused by the measurement inaccuracy and the manufacturing imperfections of the employed RFID chip.

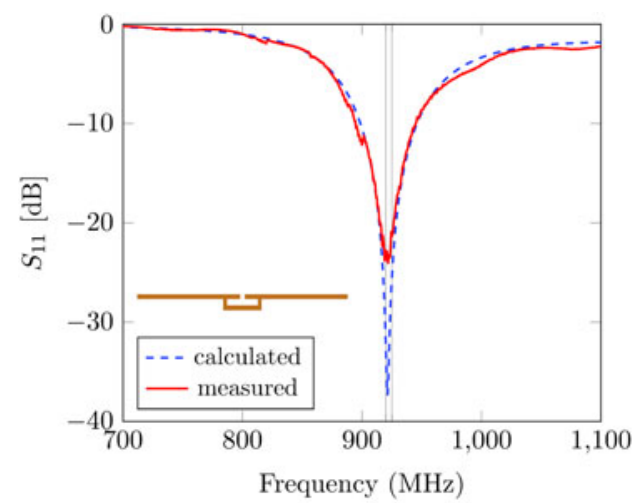

(a)

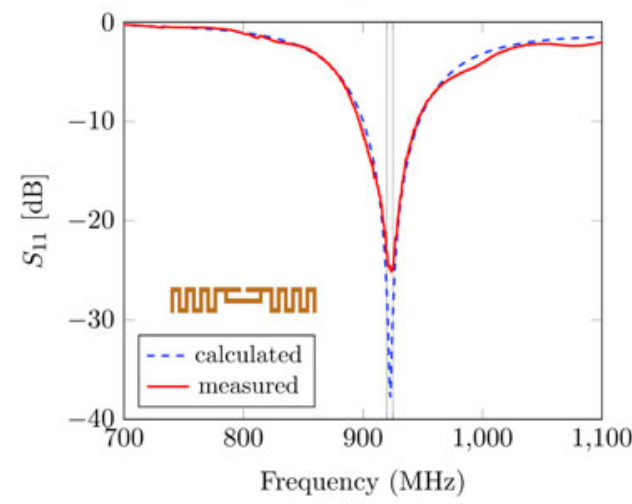

(c)
To examine the quality of impedance matching between the fabricated tag antennas and the employed RFID chip, the measured $S_{11}$ results are derived from the measured antenna impedance results and the measured chip impedance results using equation (2). The calculated $S_{11}$ results are derived from the calculated antenna impedance results and the defined chip impedance data. The calculated and measured $S_{11}$ results of the fabricated tag antennas are shown in Fig. 11. It is noted that the calculated and measured $S_{11}$ results of each antenna are in good agreement and reach the minimum point at around a frequency of $922.5 \mathrm{MHz}$. Here, the slight offsets observed between the calculated and measured $S_{11}$ results are mainly caused by the deviations between the measured and defined impedance data of the employed RFID chip. The results suggest that the proposed ink-reducing antenna designs are well-matched with the RFID chip at the designed operating frequency of 922.5 MHz. Table 3 summarizes the calculated and measured results of the impedance and $S_{11}$ of the RFID tag antennas and RFID chip at $922.5 \mathrm{MHz}$.

\section{C) Read range measurement}

The read range measurement is done inside the Micronix ME8661B anechoic chamber box as illustrated in Fig. 12. An RFID tag under test and an $8.94 \mathrm{dBi}$ linearly polarized reader antenna are placed inside the chamber box. The measured RFID tag is placed at a distance of $1 \mathrm{~m}$ from the reader antenna. The reader antenna is fed by an Alien ALR-9900 RFID reader connected to a computer located outside the

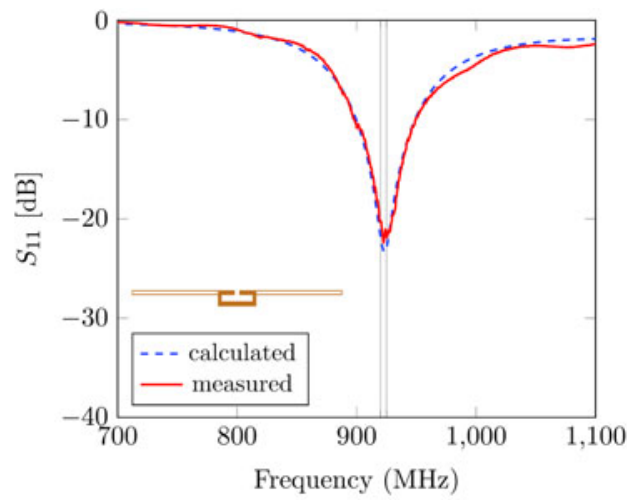

(b)

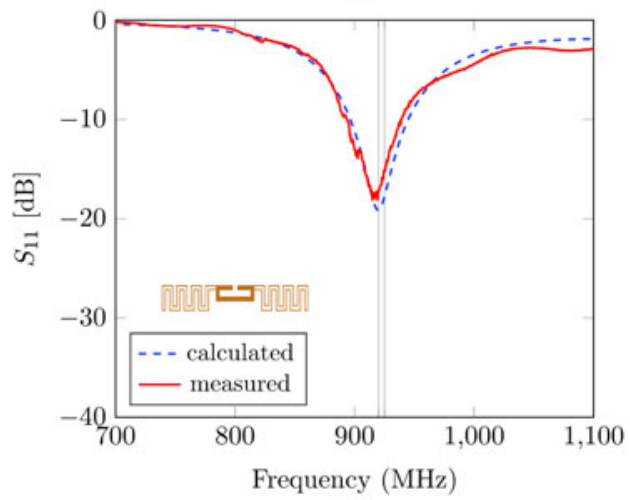

(d)

Fig. 11. Calculated and measured $S_{11}$ results of the fabricated tag antennas: (a) the $0.36 \lambda$ straight dipole antenna, (b) the ink-reducing o.36 $\lambda$ straight dipole antenna, (c) The $0.25 \lambda$ meander dipole antenna, and (d) the ink-reducing $0.25 \lambda$ meander dipole antenna. 
Table 3. Impedance and $S_{11}$ results of the RFID tag antennas and RFID chip at $922.5 \mathrm{MHz}$.

\begin{tabular}{|c|c|c|c|c|c|c|}
\hline Antenna & $\begin{array}{l}\text { Resistance } \\
\text { (calculated) }(\Omega)\end{array}$ & $\begin{array}{l}\text { Reactance } \\
\text { (calculated) }(\boldsymbol{\Omega})\end{array}$ & $\begin{array}{l}S_{11} \\
\text { (calculated) }\end{array}$ & $\begin{array}{l}\text { Resistance } \\
\text { (measured) }(\boldsymbol{\Omega})\end{array}$ & $\begin{array}{l}\text { Reactance } \\
\text { (measured) }(\Omega)\end{array}$ & $\begin{array}{l}S_{11} \\
\text { (measured) }\end{array}$ \\
\hline $0.36 \lambda$ straight dipole & 14.58 & 127.2 & $-33.9 \mathrm{~dB}$ & 20.82 & 126.1 & $-22.1 \mathrm{~dB}$ \\
\hline $\begin{array}{c}\text { Ink-reducing } 0.36 \lambda \\
\text { straight dipole }\end{array}$ & 15.99 & 126.0 & $-23.2 \mathrm{~dB}$ & 19.94 & 125.8 & $-22.4 \mathrm{~dB}$ \\
\hline $0.25 \lambda$ meander dipole & 14.41 & 126.6 & $-37.2 \mathrm{~dB}$ & 17.17 & 127.8 & $-26.2 \mathrm{~dB}$ \\
\hline $\begin{array}{c}\text { Ink-reducing } 0.25 \lambda \\
\text { meander dipole }\end{array}$ & 17.80 & 127.0 & $-18.7 \mathrm{~dB}$ & 19.83 & 131.0 & $-16.1 \mathrm{~dB}$ \\
\hline RFID chip & 14.10 & -126.9 & - & 18.80 & -128.5 & - \\
\hline
\end{tabular}

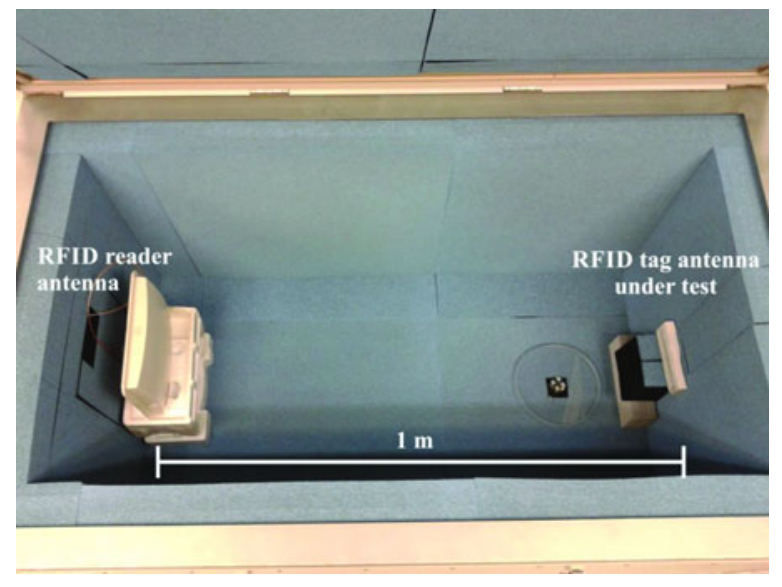

Fig. 12. Read range measurement setup inside an anechoic chamber box.

chamber box. The transmitted output power from the reader antenna is varied by adjusting the attenuation level. Then, the minimum power level at which the reader can still communicate with the RFID tag $\left(P_{\text {min }}\right)$ is recorded. The tag's read range can be determined by [25]

$$
r=d \sqrt{\frac{E I R P}{P_{\min } L g_{t}}},
$$

where $L$ is the loss of the connecting cable, $g_{t}$ is the gain of the transmitting antenna, and $d$ is the distance of the reader antenna to the tag.

Table 4 presents the calculated and measured gain, and read range results of the fabricated RFID tags. Note that the measured antenna gain results are obtained from the next section. It is noticed that the measured read range is slightly shorter than the calculated ones. The $0.36 \lambda$ straight dipole antenna delivers the read range of $6.92 \mathrm{~m}$ which is the longest read range among the fabricated designs, while the
$0.36 \lambda$ straight dipole antenna with hollowed-out arms achieves a slightly shorter read range of $6.45 \mathrm{~m}$. The $0.25 \lambda$ meander dipole antenna delivers a read range of $5.54 \mathrm{~m}$, while the optimized ink-reducing design achieves a read range of $4.71 \mathrm{~m}$. In summary, the measured results are quite consistent with the calculated results.

\section{D) Radiation pattern measurement}

The radiation pattern of the printed antenna is also measured inside the Micronix ME8661B anechoic chamber box using the Single-ended/Balanced topology of the four-port Keysight PNA-X N5245A VNA. The fabricated printed antenna is placed on a turn table in the chamber box. The measured results of the ink-reducing designs are normalized to the maximum measured gain values of the conventional fully filled antenna counterparts. The maximum values of the measured antenna gain results for the antenna designs under consideration are summarized in Table 4.

The measured and simulated radiation patterns of the $0.36 \lambda$ straight dipole antenna designs and the $0.25 \lambda$ meander dipole antenna designed are displayed in Figs 13 and 14 , respectively. The $H$ - and $E$-plane radiation patterns of the conventional and ink-reducing dipole antenna designs are compared. It can be seen that all of the printed antenna designs under consideration achieve the omni-directional radiation pattern in the $H$-plane. Furthermore, the measured and simulated radiation pattern results are quite consistent. The results confirm that the radiation patterns of the proposed ink-reducing hollowed-out arm antenna structure resemble those of the conventional solid-arm dipole antenna designs.

\section{CONCLUSION}

This paper presents an optimized dipole antenna design that can achieve good antenna performance and offers a considerable ink consumption reduction. The antenna arms of the

Table 4. Read range results of the fabricated RFID tag antennas.

\begin{tabular}{|c|c|c|c|c|c|}
\hline Antenna & $\begin{array}{l}\text { Gain (calculated) } \\
(\mathrm{dBi})\end{array}$ & $\begin{array}{l}\text { Gain (measured) } \\
(\mathrm{dBi})\end{array}$ & $\begin{array}{l}\text { Read range (calculated) } \\
(\mathrm{m})\end{array}$ & $\begin{array}{l}\text { Read range (measured) } \\
(\mathrm{m})\end{array}$ & $\begin{array}{l}\text { Ink volume } \\
\left(\mathrm{mm}^{3}\right)\end{array}$ \\
\hline $0.36 \lambda$ straight dipole & -2.63 & -3.51 & 7.01 & 6.92 & 0.288 \\
\hline $\begin{array}{l}\text { Ink-reducing } 0.36 \lambda \text { straight } \\
\text { dipole }\end{array}$ & -3.28 & -4.08 & 6.50 & 6.45 & 0.174 \\
\hline $0.25 \lambda$ meander dipole & -4.26 & -4.38 & 5.63 & 5.54 & 0.428 \\
\hline $\begin{array}{l}\text { Ink-reducing } 0.25 \lambda \text { meander } \\
\text { dipole }\end{array}$ & -4.79 & -5.07 & 5.27 & 4.71 & 0.230 \\
\hline
\end{tabular}




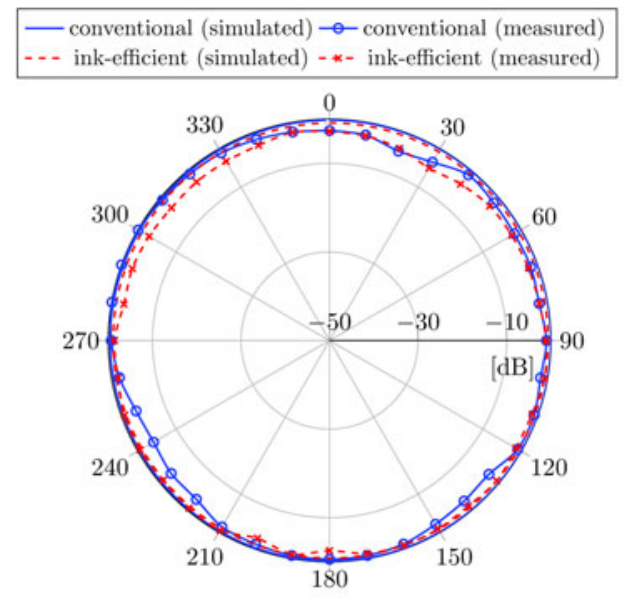

(a)

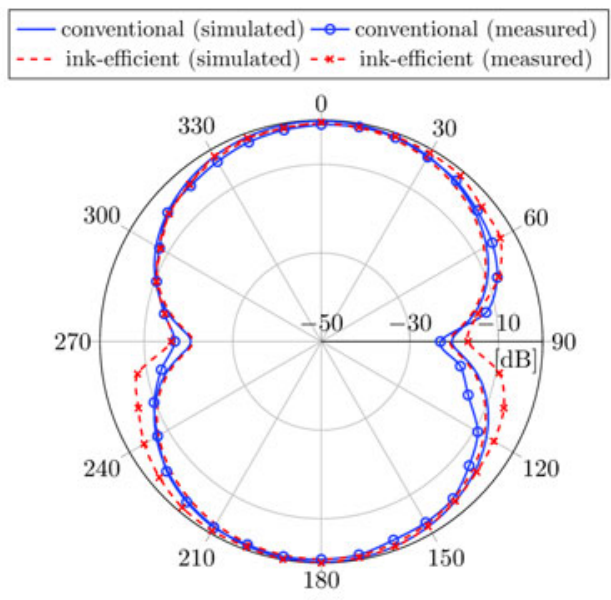

(b)

Fig. 13. Measured and simulated radiation patterns of the conventional and ink-reducing $0.36 \lambda$ straight dipole antenna designs: (a) $H$-plane and (b) $E$-plane.

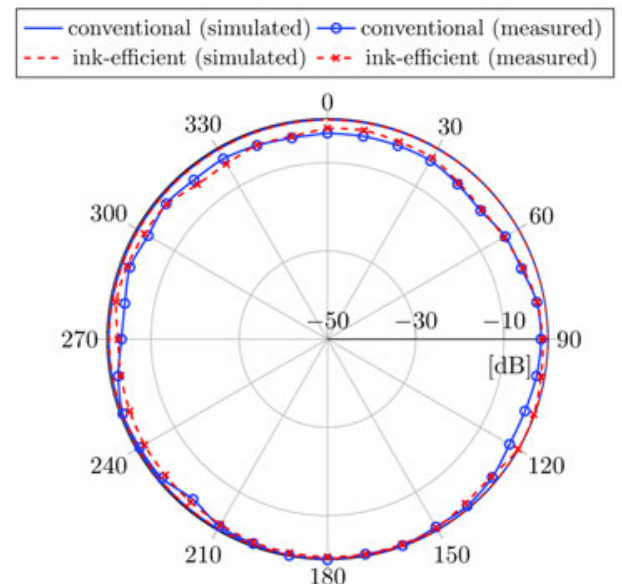

(a)

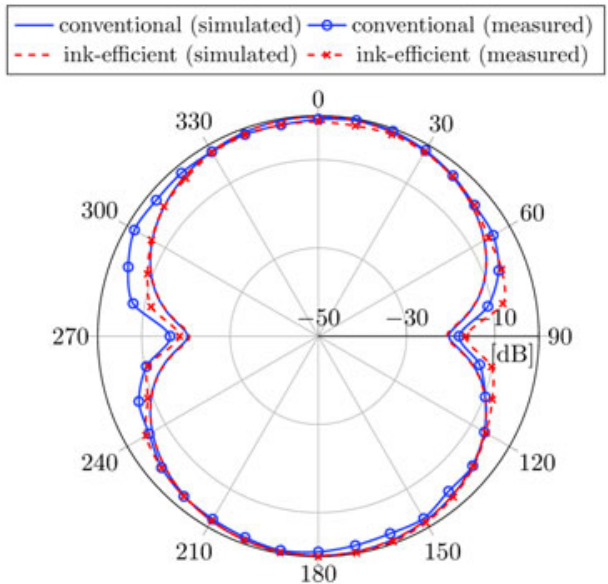

(b)

Fig. 14. Measured and simulated radiation patterns of the conventional and ink-reducing $0.25 \lambda$ meander dipole antenna designs: (a) $H$-plane and (b) $E$-plane.

proposed designs are hollowed out, so that much less ink is used to fabricate the RFID tags. The matching loop section should not contain a hollowed-out area. Otherwise, the performance of the RFID tag would drastically degrade. The proposed ink-reducing dipole antenna designs can achieve comparable performance, reduce ink consumption by almost half, and maintain the omni-directional radiation pattern over the $H$-plane. The proposed designs are fabricated using the inkjet printing technology for design verification. The measured and simulated performance results are quite consistent. For future research work, the proposed ink-reducing meander dipole antenna structure may be combined with other existing and new techniques to obtain better tag antenna designs.

\section{REFERENCES}

[1] Subramanian, V.; Frechet, J.M.J.; Chang, P.C.; Huang, D.C.; Lee, J.B.; Molesa, S.E.; Murphy, A.R.; Redinger, D.R.; Volkman, S.K.: Progress toward development of all- printed RFID tags: materials, processes, and devices. Proc. IEEE, 93 (7) (2005), 1330-1338.
[2] Pranonsatit, S.; Worasawate, D.; Sritanavut, P.: Affordable ink-jet printed antennas for RFID applications. IEEE Trans Compon. Packag. Manuf. Technol., 2 (2012), 878-883.

[3] Mantysalo, M.; Pekkanen, V.; Kaija, K.; Niittynen, J.; Koskinen, S.; Halonen, E.; Mansikkamaki, P.; Hameenoja, O.: Capability of inkjet technology in electronics manufacturing, in 59th Electronic Components and Technology Conf., IEEE, 2009, 1330-1336.

[4] Bjorninen, T.; Merilampi, S.; Ukkonen, L.; Sydanheimo, L.; Ruuskanen, P.: The effect of fabrication method on passive UHF RFID tag performance. Int. J. Antennas Propag., (2009), 1-8.

[5] Rida, A.; Yang, L.; Vyas, R.; Tentzeris, M.M.: Conductive inkjetprinted antennas on flexible low-cost paper-based substrates for RFID and WSN applications. IEEE Antennas Propag. Mag., 51 (2009), 13-23.

[6] Yang, L.; Rida, A.; Vyas, R.; Tentzeris, M.M.: RFID tag and RF structures on a paper substrate using inkjet-printing technology. IEEE Trans. Microw. Theory Tech., 55 (12) (2007), 2894-2901.

[7] Merilampi, S.; Ukkonen, L.; Sydanheimo, L.; Ruuskanen, P.; Kivikoski, M.: Analysis of silver ink bow-tie RFID tag antennas printed on paper substrates. Int. J. Antennas Propag., 2007 (2007), 1-9.

[8] Siden, J.; Olsson, T.; Koptioug, A.; Nilsson, H.E.: Reduced amount of conductive ink with gridded printed antennas, in 5 th Int. Conf. on 
Polymers and Adhesives in Microelectronics and Photonics, IEEE, 2005, 86-89.

[9] Bentaher, K.; Choubani, F.; Vuong, T.H.; David, J.: Design of nonuniform meander line antennas for passive RFID tags in the UHF band, in PIERS Proc., 2011, 400-403.

[10] Virtanen, J.; Bjorninen, T.; Ukkonen, L.; Sydanheimo, L.: Passive UHF inkjet-printed narrow-line RFID tags. IEEE Antennas Wireless Propag. Lett., 9 (2010), 440-443.

[11] Siden, J.; Fein, M.K.; Koptyug, A.; Nilsson, H.E.: Printed antennas with variable conductive ink layer thickness. IET Microw. Antennas Propag., 1 (2) (2007), 401-407.

[12] Pynttari, V.; Halonen, E.; Sillanpaa, H.; Mantysalo, M.; Makinen, R.: $\mathrm{RF}$ design for inkjet technology: antenna geometries and layer thickness optimization. IEEE Antennas Wireless Propag. Lett., 11 (2012), 188-191.

[13] Virtanen, J.; Virkki, J.; Elsherbeni, A.Z.; Sydanheimo, L.; Ukkonen, L.: A selective ink deposition method for the cost-performance optimization of inkjet-printed UHF RFID tag antennas. Int. J. Antennas Propag., 2012 (2012), 1-9.

[14] Orecchini, G.; Alimenti, F.; Palazzari, V.; Rida, A.; Tentzeris, M.M.; Roselli, L.: Design and fabrication of ultra-low cost radio frequency identification antennas and tags exploiting paper substrates and inkjet printing technology. RF/Microw. Commun. Subsyst. Emerging Wireless Technol. (Spec. Issue), 5 (8) (2011), 993-1001.

[15] Toccafondi, A.; Giovampaola, C.D.; Braconi, P.; Cucini, A.: UHF RFID integrated transponder for moving vehicle identification. Appl. Comput. Electromagn. Soc. J. (Spec. Issue on Comput. Exp. Tech. RFID Syst. Appl.), 25 (6) (2010), 543-551.

[16] Nikitin, P.V.; Rao, K.V.S.; Lam, S.F.; Pillai, V.; Martinez, R.; Heinrich, H.: Power reflection coefficient analysis for complex impedances in RFID tag design. IEEE Trans. Microw. Theory Tech., 53 (9) (2005), 2721-2725.

[17] Balanis, C.A.: Antenna Theory: Analysis and Design, Wiley, New York, 1997.

[18] Nakano, H.; Tagami, H.; Yoshizawa, A.; Yamauchi, J.: Shortening ratios of modified dipole antennas. IEEE Trans. Antennas Propag., 32 (4) (1984), 385-386.

[19] Marrocco, G.; Fonte, A.; Bardati, F.: Evolutionary design of miniaturized meander-line antennas for RFID applications, in IEEE Antennas and Propagation Society Int. Symp., vol. 2, 2002, 362-365.

[20] Kellomaki, T.; Virkki, J.; Merilampi, S.; Ukkonen, L.: Towards washable wearable antennas: a comparison of coating materials for screen-printed textile-based UHF RFID tags. Int. J. Antennas Propag., 2012 (2012), 1-11.

[21] Scarpello, M.L.; Kazani, I.; Hertleer, C.; Rogier, H.; Ginste, D.V.: Stability and efficiency of screen-printed wearable and washable antennas. IEEE Antennas Wireless Propag. Lett., 11 (2012), 838-841.

[22] Palmer, K.D.; van Rooyen, M.W.: Simple broadband measurements of balanced loads using a network analyzer. IEEE Trans. Instrum. Meas., 55 (1) (2006), 266-272.

[23] Kuo, S.K.; Chen, S.L.; Lin, C.T.: An accurate method for impedance measurement of RFID tag antenna. Progr. Electromagn. Res., 83 (2008), 93-106.

[24] Qing, X.; Goh, C.K.; Chen, Z.N.: Impedance characterization of RFID tag antennas and application in tag co-design. IEEE Trans. Microw. Theory Tech., 57 (5) (2009), 1268-1274.

[25] Rao, K.V.S.; Nikitin, P.V.; Lam, S.F.: Antenna design for UHF RFID tags: a review and a practical application. IEEE Trans. Antenna Propag., 53 (2005), 3870-3876.

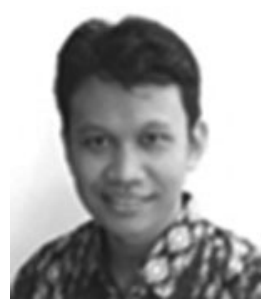

Adi M. J. Marindra received a degree in Electrical Engineering from Gadjah Mada University, Indonesia, in 2010 and received a master's degree in Computer Engineering from King Mongkut's Institute of Technology Ladkrabang, Thailand, in 2013. He is currently a research assistant in RF Electronics Laboratory at the National Electronics and Computer Technology Center (NECTEC), Thailand. His main research interests are wireless communications, antennas, and radio propagation.

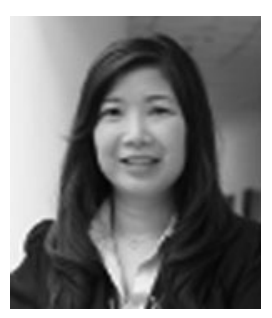

Pornanong Pongpaibool received her B.E. degree in Electrical Engineering from Chulalongkorn University, Thailand, in 1999, and got the Japanese Government Scholarship to pursue graduate studies in Japan. She received her M.Eng. degree in System Engineering from Shizuoka University in 2002 and D.Eng. degree in Electronic and Information Engineering from Tokyo University of Agriculture and Technology in 2005. After her graduation, she joined the National Institute of Information and Communications Technology (NICT), Japan, from April 2005 to March 2007. She is currently with the National Electronics and Computer Technology Center (NECTEC), Thailand. Her research interests are antennas, RFID, computational electromagnetic, and printed electronics. Dr. Pornanong is currently a Lab Head of RF Electronics Laboratory, Wireless Innovation and Security Research Unit, NECTEC. She received the 2004 IEEE AP-S Japan Chapter Young Engineer Award and 2004 IEICE Young Researcher's Award.

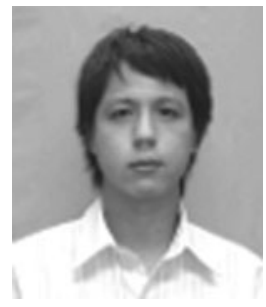

Werayuth Wallada received his B.Ind.Tech. degree in Electronics \& Computer Technology from King Mongkut's University of Technology North Bangkok, Thailand, in 2008. He is currently a research assistant in RF Electronics Laboratory at the National Electronics and Computer Technology Center (NECTEC), Thailand. His research interests are electronic circuits, RFID, and antennas.

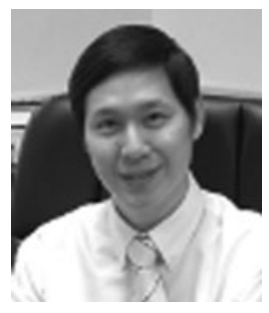

Siwaruk Siwamogsatham received his B.S.E.E. degree from Chulalongkorn University, Thailand in 1994, and earned a scholarship from the Thai Government to pursue graduate studies in the USA. He received his M.S. and Ph.D. degrees in the field of wireless communications and networking from The Ohio State University, USA, in 1997 and 2002, respectively. After his graduation, he joined the National Electronics and Computer Technology Center (NECTEC), Thailand, where he has focused his research interest in the field of wireless and security technologies. His 
highlighted $\mathrm{R} \& \mathrm{D}$ contributions include efficient printed antenna designs for RFID applications, ultra-low standby power consumption systems, wireless energy harvesting, ubiquitous and context-aware systems, secure wireless LAN systems, and wireless communication devices for national security applications. Dr. Siwaruk is currently a Deputy Executive Director at NECTEC. 